\title{
Multilevel Current Source Inverter to Improve Power Quality in a Distribution Network
}

\author{
Miguel Aguirre ${ }^{1}$ \\ Laura Calviño \\ CIDEI - ITBA \\ Instituto Tecnológico de Buenos Aires \\ Buenos Aires, Argentina \\ maguir@itba.edu.ar_ lcalvino@alu.itba.edu.ar
}

\author{
V. Fabián Corasaniti María Inés Valla ${ }^{2}$ \\ IITREE-LAT and LEICI - Facultad de Ingeniería \\ Universidad Nacional de La Plata \\ La Plata, Argentina \\ v.f.corasaniti@ieee.org \\ m.i.valla@ieee.org
}

\begin{abstract}
This paper deals with the problem of reactive power and harmonics in a standard medium voltage (MV) distribution network. It presents the design of a shunt active filter implemented with a multilevel current source inverter (MCSI) connected to the medium voltage level of a power distribution system. The proposed MCSI is made by identical modules where all inductors carry the same amount of current. The current balance is achieved by a Phase-Shifted Carrier SPWM proper implementation. The performance of proposed active filter is thoroughly simulated with Matlab Simulink. It shows very good behavior in steady state and transient conditions.
\end{abstract}

\section{INTRODUCTION}

Power quality in distribution systems is seriously affected by harmonics introduced by the loads. Power factor is another issue to deal with when maximum efficiency of the system is pursued. Both problems can be solved in a medium voltage distribution system by adding a shunt active filter based on a Multilevel Current Source Inverter (MCSI).

Multilevel voltage source inverters (MVSI) have been used in power factor correction and active filters applications in recent years [1], but they require high voltage and high ripple current capacitors [2] whose life spam and stability are worst than those for inductors, leading to a higher failure ratio. Inductors might be less volume effective than capacitors but they have a much longer cycle life. They can stand high voltage ripple without losing performance and their electrical attributes hardly change with time, as long as good power dissipation is provided. This means higher reliability, larger mean time between faults (MTBF) and less maintenance needs. Inductors built with high temperature superconductors will reduce losses, turning MCSI into the most efficient solution for multilevel inverters [3].

Multilevel topologies present several advantages in both VSI and CSI structures, regarding total harmonic distortion and stress on components and switches [2] [4]. Nevertheless they have not yet been widely applied, the MCSI appears as a smart choice to improve performance and efficiency in medium voltage distribution systems and industrial applications where high power or high current are required, such as active filters, motor drives and HVDC [5].

In this paper a single-rating-inductor-MCSI is employed to

${ }^{1} \mathrm{M}$. Aguirre is currently working toward the Doctor in Engineering degree at Universidad Nacional de La Plata (UNLP).

${ }^{2} \mathrm{M}$. Valla is also with CONICET, Argentina implement an active filter [6]. The converter consists of three identical modules which generate seven current levels [7]. Each module uses two balance inductors and six power switches. Both inductors of every module should carry the same current. The current flowing through the inductors can be balanced when applying a state machine modulation that properly uses the redundant zero states [8][9]. Moreover the switching frequency can be reduced with this modulation. The modulation and gate drives control logic are implemented on a Field-Programmable-Gate-Array (FPGA) [10], which is a powerful, cost-effective solution. It allows complex logic and control algorithms, fast speed and multiple $\mathrm{I} / \mathrm{O}$ pins, which becomes especially attractive for multilevel converters control.

Fault tolerant assembles are easy to develop and operate because all modules are identical. Fault tolerant capabilities are a mayor feature in power quality increasing reliability and reducing MTBF. They can be achieved by adding hot spare modules and have been already demonstrated for the converter in [11].

This paper presents a simple approach, showing that a good power factor correction performance and harmonic current minimization can be provided. Current balance among modules is sustained by adapting a well known PWM strategy [9] while minimizing switching speed using a sequential machine approach. In detail, the paper is organized as follows. The system is described in Section II. The active filter is analyzed in Section III, including a comprehensive analysis of the inverter in section III A and the control scheme in section III B. The performance of the proposed filter is evaluated in section IV with simulations. Finally, some conclusions are drawn in Section V.

\section{SYSTEM DESCRIPTION}

The one line diagram of the system model adopted for the medium power distribution system under test is shown in Fig.1. The loads of the different substations are mainly commercial and residential, so it is more difficult to identify the harmonic sources than in the case of industrial plants. Then, it is necessary to build a model based on the field measurements [12].

The system is represented as an ideal voltage source of $132 \mathrm{kV}$ connected to three transformers of similar characteristics, $132 / 34.5 / 13.8 \mathrm{kV}$ and $15 / 10 / 15 \mathrm{MVA}$. The system is modeled by its equivalent impedance related to 